\title{
Contribuição ao protocolo de monitoramento ambiental da maricultura de Kappaphycus alvarezii (Doty) Doty ex P.C. Silva (Areschougiaceae - Rhodophyta) na baía de Sepetiba, RJ, Brasil
}

\author{
Beatriz Castelar ${ }^{1,3}$, Renata Perpetuo Reis ${ }^{1}$ e Marcos Bastos ${ }^{2}$
}

Recebido em 3/01/2008. Aceito em 11/09/2008

\begin{abstract}
RESUMO - (Contribuição ao protocolo de monitoramento ambiental da maricultura de Kappaphycus alvarezii (Doty) Doty ex P.C. Silva (Areschougiaceae - Rhodophyta) na baía de Sepetiba, RJ, Brasil). Kappaphycus alvarezii, uma das principais fontes de matéria prima para produção de carragenana, vem sendo cultivada em balsas flutuantes, na baía de Sepetiba, sem estudos de monitoramento ambiental. A ocorrência e o estabelecimento de esporos e de mudas desta alga exótica no ambiente marinho foram avaliados e a praticidade da metodologia aplicada foi verificada, visando à proposta de um protocolo de monitoramento ambiental para cultivos liberados para o Brasil, entre a baía de Sepetiba (RJ) e a Ilha Bela (SP). Este estudo foi realizado no maior cultivo desta espécie no país, na baía de Sepetiba (2303'50"S e $\left.043^{\circ} 52^{\prime} 50^{\prime} \mathrm{N}\right)$, entre maio e julho/2006. Neste período, não foi observada ocorrência de mudas férteis sob cultivo tampouco estabelecimento de esporos. A ocorrência de mudas fora do sistema de cultivo foi ínfima, frente à biomassa cultivada, e restrita aos limites da fazenda marinha. Além disso, a sobrevivência de mudas transplantadas fora das balsas foi suprimida em detrimento da baixa incidência luminosa provocada pelo alto teor de material particulado em suspensão na água do mar. Concluiu-se, portanto, que a introdução de Kappaphycus alvarezii não ocasionou danos ambientais nesta baía até o momento e que o método utilizado foi adequado à avaliação do estabelecimento desta espécie no ambiente. Neste sentido, recomenda-se a aplicação permanente do método apresentado como protocolo para o cultivo de Kappaphycus alvarezii na área permitida para o Brasil, visando à manutenção da biodiversidade marinha.
\end{abstract}

Palavras-chave: alga exótica, maricultura de algas, introdução de espécies

\begin{abstract}
Contribution to the environmental monitoring protocol for Kappaphycus alvarezii (Doty) Doty ex P.C. Silva (Areschougiaceae - Rhodophyta) cultivation at Sepetiba bay, Rio de Janeiro State, Brazil). Kappaphycus alvarezii, one of the most important sources of raw material for carrageenan production, has been cultivated in a floating-raft system, at Sepetiba Bay, southeast Brazil, with no environmental monitoring studies. The aim of this research was to verify the occurrence and establishment of Kappaphycus alvarezii plantlets and spores in

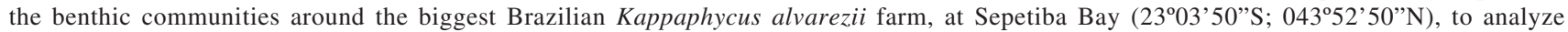
the applicability of the methodology and to propose an environmental monitoring protocol for areas where this cultivation is permitted in Brazil. From May to July/2006, the occurrence of fertile cultivated branches as well the recruitment and establishment of spores were not observed. The occurrence of plantlets outside the system was restricted to the farm boundaries and lost biomass was insignificant in relation to cultivated biomass. Futhermore, the establishment of transplanted seedlings through vegetative growth was suppressed by high suspended particulate material in the water column. These results suggest that Kappaphycus alvarezii introduction has not yet provoked environmental damage in Sepetiba Bay and the methodology used was adequate for this kind of evaluation. Constant application of the method is recommended as a protocol for growing Kappaphycus alvarezii where permitted in Brazil in order to maintain marine biodiversity.
\end{abstract}

Key words: exotic seaweed, seaweed cultivation, species introduction

\section{Introdução}

A demanda mundial de matéria prima para a produção de carragenana é crescente (Ask \& Azanza 2002) sendo estimado um aumento anual de cerca de $5 \%$ para as próximas décadas (Eklöf et al. 2005). No Brasil, existem duas fontes de matéria prima para produção de carragenana, uma a partir do cultivo da alga exótica, Kappaphycus alvarezii (Doty) Doty ex P.C. Silva (Areschougiaceae - Rhodophyta), e outra a partir da alga nativa, Hypnea musciformis (Wulfen) J.V. Lamouroux (Cystocloneaceae - Rhodophyta), coletada em bancos naturais (Paula et al. 1998). Apesar de H. musciformis apresentar ampla distribuição na costa brasileira, sua coleta é insuficiente para suprir a demanda nacional. Além disso, ainda que sua taxa de crescimento atinja até $10 \% \cdot$ dia $^{-1}$ (Reis et al. 2006), esta espécie apresenta difícil adaptação às tecnologias de cultivo (Oliveira \& Miranda 1998).

A introdução de Kappaphycus alvarezii no Brasil foi proposta com intuito de minimizar a explotação dos bancos naturais de $H$. musciformis e de diminuir a dependência da matéria prima importada (Oliveira 1990; Paula et al. 2002). Sendo assim, em 1995 iniciou-se um programa experimental de introdução desta espécie no país, desenvolvido em Ubatuba, SP, Brasil (Paula et al. 1998) e em 2004 foi iniciado o primeiro cultivo comercial de $K$. alvarezii na baía de Sepetiba, RJ, Brasil.

Contudo, danos ambientais causados pela introdução intencional de espécies têm sido amplamente relatados (FAO 1996; CBD 2004). De acordo com 'International Union for the Conservation of Nature - IUCN', a introdução de espécies é uma das principais responsáveis pela perda da biodiversidade. Os danos ambientais sobre os recifes coralíneos havaianos causados pela introdução intencional de espécies de Kappaphycus/Eucheuma são os mais bem documentados (Conklin \& Smith 2005). Vinte e cinco anos após a introdução na baía de Kane'ohe, no Havaí, essas algas se estabeleceram sobre os corais, causando-lhes a morte principalmente por sombreamento, e continua se dispersando para outras regiões (Conklin \& Smith 2005). Também foram relatados estabelecimento de K. alvarezii

\footnotetext{
1 Instituto de Pesquisas Jardim Botânico do Rio de Janeiro, Diretoria de Pesquisa Científica, Rio de Janeiro, RJ, Brasil

2 Universidade do Estado do Rio de Janeiro, Faculdade de Oceanografia, Rio de Janeiro, RJ, Brasil

3 Autor para correspondência: beatrixcastelar@gmail.com
} 
em recifes na Índia (Bagla 2008; Chandrasekaran et al. 2008) e na Venezuela (Barrios et al. 2007).

Para evitar danos ambientais causados pela introdução intencional de algas é necessária à implementação de procedimentos de avaliação de risco e/ou de monitoramento ambiental para verificar tanto o estabelecimento da espécie introduzida no meio ambiente quanto à introdução de organismos indesejados associados à espécie-alvo (Oliveira 2005; Verlecar \& Pereira 2006). Entretanto, são incipientes os programas de monitoramento ambiental das introduções intencionais de espécies de Kappaphycus no mundo (Ask et al. 2003; Conklin \& Smith 2005).

No Brasil, de acordo com a Instrução Normativa n. 185 de 23 de julho de 2008, passa a ser exigida pelo Instituto Brasileiro do Meio Ambiente e dos Recursos Naturais Renováveis (IBAMA/MMA) a execução do monitoramento ambiental relativo à produção de Kappaphycus alvarezii. Desta maneira, torna-se emergente a criação de metodologias eficientes para a avaliação do estabelecimento desta alga fora das estruturas de cultivo.

Os objetivos deste estudo foram: 1) analisar a ocorrência e a viabilidade de esporos e mudas de Kappaphycus alvarezii no ambiente natural no entorno do maior do cultivo desta espécie no país e; 2) propor um protocolo de monitoramento ambiental para a maricultura de K. alvarezii na região sudeste brasileira através da avaliação da ocorrência e do estabelecimento de esporos e mudas desta espécie no entorno do maior cultivo do país.

\section{Material e métodos}

Este estudo foi conduzido de maio a julho de 2006, na baía da Marambaia (230' $50^{\prime \prime}$ 'S e $\left.43^{\circ} 52^{\prime} 50^{\prime \prime} \mathrm{N}\right)$, situada na porção sudoeste da baía de Sepetiba, litoral sul do Estado do Rio de Janeiro, Brasil (Fig. 1). A região encontra-se nas proximidades da principal conexão da baía de Sepetiba com o Oceano Atlântico, onde ocorrem as correntes de maior intensidade (superior a $50 \mathrm{~cm} \cdot \mathrm{s}^{-1}$ ), além de um volume maior de águas renovadas (Signorini 1980; SEMADS 2001). A baía da Marambaia possui uma profundidade média de 2,3 m, é limitada a leste por um cordão arenoso de cerca de $2 \mathrm{~km}$ de extensão e a oeste pela Ilha da Marambaia, sendo composta estritamente por substratos não consolidados de frações granulométricas predominantemente finas do tipo lodoso (Pereira et al. 2003).

O cultivo de Kappaphycus alvarezii foi instalado em 2004, a aproximadamente $60 \mathrm{~m}$ da praia, composto por 70 unidades de produção, denominadas balsas flutuantes. Cada balsa é composta por 30 subunidades, os módulos $(3 \times 5 \mathrm{~m})$, contendo 220 mudas cada um. Sob cada balsa é, ainda, instalada uma rede de nylon (60 $\mathrm{mm}$ de malha) que visa minimizar a dispersão de mudas para o ambiente natural.

A temperatura e a salinidade da água do mar foram registradas diariamente com auxílio de termômetro de mercúrio e refratômetro portátil, respectivamente. A transparência da água foi estimada com auxílio de disco de Secchi, semanalmente. Coletas de água do mar foram realizadas, também semanalmente, para a quantificação do material particulado em suspensão (MPS) e para análise da concentração de amônia, nitrito, nitrato e fosfato (Grasshoff et al. 1983). Todas as medições foram realizadas em triplicatas.

A ocorrência de material fértil (tetrásporos e carpósporos) foi verificada através da análise de 135 mudas de Kappaphycus alvarezii retiradas aleatoriamente (tabela de números aleatórios) das balsas flutuantes. Os talos foram observados em campo com auxílio de lupa manual. Cortes anatômicos, realizados à mão livre, foram observados através de microscopia óptica em laboratório. Para observar a fixação de esporos liberados, 36 placas de acrílico $(10 \times 10 \mathrm{~cm})$, com ranhuras na face superior, foram aleatoriamente instaladas sob as estruturas de cultivo. Metade das placas foi retirada após 20 dias, no meio do ciclo de produção comercial do cultivo, e a outra metade após 40 dias, no final do ciclo de produção comercial. Logo após a retirada, as placas foram acondicionadas em formaldeído a $4 \%$ e analisadas através de microscopia óptica a fim de se quantificar os esporos fixados.

Com o intuito de verificar o recrutamento, nove placas de acrílico, iguais às anteriores, foram aleatoriamente instaladas sob o cultivo e recolhidas após 20 dias. Após este período, as placas foram transportadas em caixa térmica com água do mar para até o Laboratório de Cultivo do Instituto de Pesquisas Jardim Botânico do Rio de Janeiro, onde o cultivo foi realizado sob condições ambientais próximas ao ideal requerido pela espécie (Paula et al. 1999; Paula et al. 2001; Paula \& Pereira 2003). O cultivo in vitro foi realizado em aquários contendo $1 \mathrm{~L}$ de água do mar filtrada em membrana de éster celulose $(0,45 \mu \mathrm{m})$, enriquecida com solução Provasoli modificada ES/4 (Starr \& Zeikus 1993), aerada por bombas de aeração de aquários e trocada a cada cinco dias. A temperatura da água foi mantida em $22 \pm 2{ }^{\circ} \mathrm{C}$, salinidade de $35 \pm 1$ e fotoperíodo de 12 horas luz. A iluminação foi obtida através de lâmpadas fluorescentes de $20 \mathrm{~W}$, sob irradiância de $130 \pm 20 \mu \mathrm{mol} \cdot \mathrm{m}^{-2} \cdot \mathrm{s}^{-1}$. Após 20 dias sob cultivo in vitro as placas de acrílico foram observadas através de microscopia óptica a fim de se quantificar, através do percentual de cobertura, o desenvolvimento das plântulas.

A ocorrência de mudas de Kappaphycus alvarezii desprendidas, fora do sistema de cultivo, foi verificada em toda a extensão da praia da baía da Marambaia e quantificada, em doze estações de coleta préestabelecidas (Fig. 1). Em cada estação, foram dispostos aleatoriamente dez quadrados amostrais $(50 \times 50 \mathrm{~cm})$ sobre um transecto de $20 \mathrm{~m}$ paralelo à linha da água e aplicada a metodologia de amostragem destrutiva adaptada de De Wreede (1985), coletando-se apenas os exemplares de Kappaphycus alvarezii. As mudas coletadas foram secas em estufa a $60{ }^{\circ} \mathrm{C}$ até obtenção de massa constante. A biomassa total desprendida do cultivo foi estimada e comparada em termos percentuais com a biomassa média total da fazenda.

Finalmente, para avaliar o estabelecimento de mudas de Kappaphycus alvarezii no ambiente natural foi utilizado um sistema de transplante. Dezoito mudas, com massa úmida inicial de $100 \mathrm{~g}$, foram atadas a blocos de cimento, instalados no limite superior da zona infralitorânea, com o intuito de simular o ambiente mais propício para o estabelecimento desta alga na baía da Marambaia. As mudas foram pesadas a cada 20 dias e o sucesso de estabelecimento avaliado através da taxa de crescimento diário expressa em \%.dia ${ }^{-1}$ e obtida

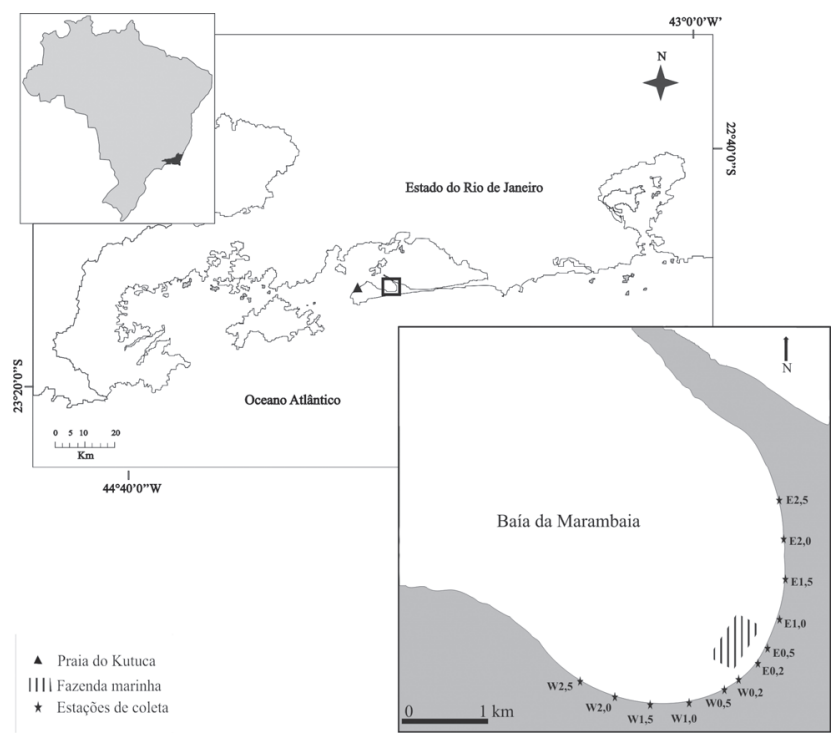

Figura 1. Mapa da baía da Marambaia, inserida na porção sudoeste da baía de Sepetiba, litoral sul do Estado do Rio de Janeiro, Brasil. 
através da fórmula: $\mathrm{TCD}=100 \times\left[\mathrm{LN}\left(\mathrm{M}_{\mathrm{f}}\right) /\left(\mathrm{M}_{\mathrm{i}}\right)\right] / \mathrm{t}$, onde $\mathrm{M}_{\mathrm{f}}=$ massa úmida final; $\mathrm{M}_{\mathrm{i}}=$ massa úmida inicial; $\mathrm{e} \mathrm{t}=$ dias de experimento (Ask 2006).

Para o tratamento estatístico, foi utilizado o pacote estatístico Statistica 6.0 StatSoft ${ }^{\circledR}$. Os dados não apresentaram normalidade (teste de Shapiro Wilk's) e/ou homogeneidade de variâncias (Teste de Cochran), portanto foram submetidos aos testes não-paramétricos (Zar 1996). As medianas das biomassas fora do sistema de cultivo nas diferentes estações de coleta foram comparadas através do teste Kruskal-Wallis e as medianas das taxas de crescimento das mudas transplantadas nos períodos de 20 e 40 dias foram comparadas pela análise de Wilcoxon. O intervalo de confiança para os testes de significância foi de $95 \%(p=0,05)$.

\section{Resultados e discussão}

As mudas de Kappaphycus alvarezii cultivadas na baía da Marambaia não se mostraram férteis durante o período estudado, uma vez que tetrásporos ou cistocarpos não foram observados nos 135 talos vistoriados. Portanto, não foi observado recrutamento de esporos nas placas acrílicas mantidas sob cultivo. Segundo Bulboa et al. (2008), após 20 dias, plântulas cultivadas in vitro alcançaram cerca de $40 \mu \mathrm{m}$ de diâmetro, porém, assim como no presente estudo, não foi registrada a ocorrência de recrutamento in situ. Desta maneira, não foi constatado o estabelecimento da espécie exótica via estruturas reprodutivas no ambiente natural na baía da Marambaia. Este resultado está de acordo com os resultados de Azanza-Corrales et al. (1992). Segundo estes autores, esta espécie geralmente apresenta-se infértil em sistemas de cultivo, ou as fases reprodutivas não ocorrem simultaneamente, sugerindo que o ciclo não se completa nos cultivos. Paula et al. (1999) reportaram mudas férteis de Kappaphycus alvarezii de dezembro de 1995 a maio de 1996, contudo, os esporos foram pouco viáveis, não apresentando, como neste estudo, potencial de estabelecimento no ambiente.

Quanto à ocorrência de mudas fora do sistema de cultivo na baía da Marambaia, foram observadas mudas em apenas um terço das estações de coleta (W0,5; W0,2; E0,2 e E0,5) e restritas à área de manejo da fazenda marinha (Fig. 1,2). A variação da biomassa das mudas foi altamente significativa entre as doze estações de coleta $\left(\mathrm{H}_{11,120}=79,68 ; p<0,001\right)$, variando de zero a 237,09 g.m $\mathrm{m}^{-2}$ (massa seca). A biomassa total de Kappaphycus alvarezii fora do sistema de cultivo, com base na amostragem realizada, foi estimada em torno de $50 \mathrm{~kg}$ de massa seca, representando $0,1 \%$ da estimativa da biomassa total cultivada na fazenda marinha. A diminuta e restrita ocorrência de Kappaphycus alvarezii no ambiente foi atribuída à baixa capacidade de estabelecimento desta alga no ambiente natural da baía da Marambaia.

Fora dos limites da fazenda, Reis (dados não publicados) constatou a ausência de Kappaphycus alvarezii no costão oeste da Praia do Kutuca (Fig. 1). Esta serve como uma área-controle estratégica para o monitoramento ambiental deste cultivo, pois está localizada a cerca de $20 \mathrm{~km}$ a oeste da baía da Marambaia, ou seja, fora da área de abrangência da fazenda marinha, além de estar sob influência de um dos canais mais importantes de entrada de correntes da baía de

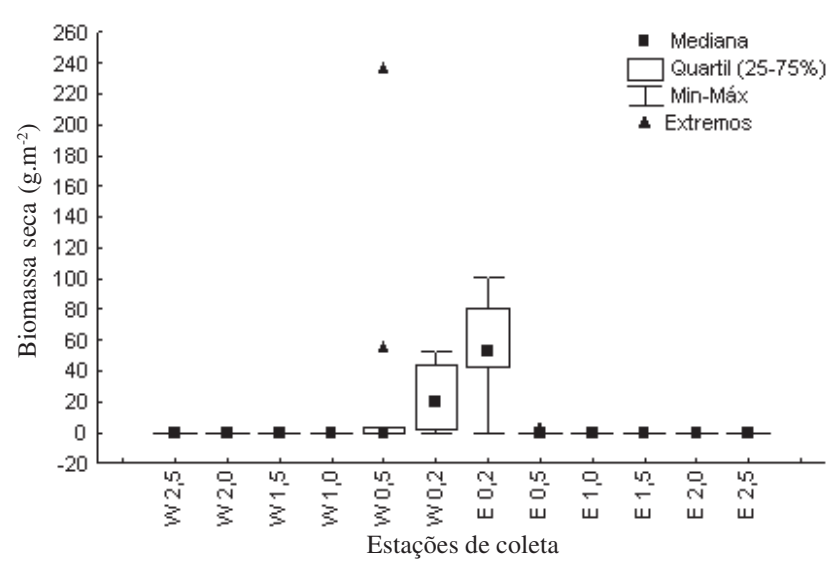

Figura 2. Variação da biomassa seca $\left(\right.$ g.m $\left.\mathrm{m}^{-2}\right)$ de mudas de Kappaphycus alvarezii (Doty) Doty ex P.C. Silva nas estações de coleta ao longo da praia da baía da Marambaia, RJ, Brasil, em junho/2006.

Sepetiba (SEMADS 2001) e da comunidade algácea local estar sendo monitorada desde 1999, existindo, portanto, dados pretéritos à instalação da fazenda realizados por Amado Filho et al. (2003).

As taxas de crescimento diário (TCD) das mudas de Kappaphycus alvarezii transplantadas apresentaram valores negativos $\left(-14,21 \pm 18,0 \% \cdot\right.$ dia $\left.^{-1}\right)$ e não diferiram entre os períodos avaliados $(Z=1,24 ; p=0,21$; Fig. 3$)$. Todas as mudas transplantadas morreram antes de 60 dias de experimento. Possivelmente, o estabelecimento das mudas foi limitado pela luz, uma vez que a estimativa da transparência da água do mar, associada ao elevado teor de material particulado em suspensão propiciaram uma baixa incidência luminosa durante todo o período experimental (Tab.1). Kappaphycus alvarezii é considerada tolerante à turbidez, desde que em baixa freqüência (Mshigeni 1979; Doty 1987; Trono 1993). Ressalta-se que no período amostrado, a temperatura superficial da água do mar e a salinidade se mantiveram dentro dos limites adequados ao crescimento de Kappaphycus alvarezii (Tab. 1; Glenn \& Doty 1990; Paula $\&$ Pereira 2003; Bulboa \& Paula 2005) e que os nutrientes apresentaram valores superiores aos requeridos pela espécie (Tab. 1), sendo Kappaphycus alvarezii conhecida

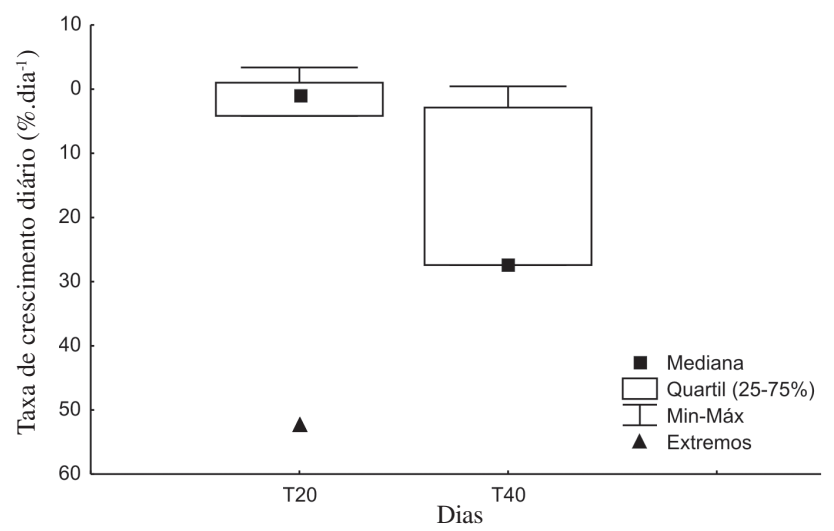

Figura 3. Taxa de crescimento diário das mudas de Kappaphycus alvarezii (Doty) Doty ex P.C. Silva transplantadas na baía da Marambaia, pesadas após 20 dias (T20) e 40 dias (T40) entre maio e julho/2006, RJ, Brasil. 
por seu baixo requerimento de nitrogênio e fósforo (Wakibia et al. 2006).

Kappaphycus alvarezii não se estabeleceu no ambiente natural via estruturas reprodutivas ou via crescimento vegetativo de mudas desprendidas do cultivo, sendo a restrição luminosa decorrente do alto teor de material particulado em suspensão, um dos principais fatores

Tabela 1. Valores (mínimos) medianos (máximos) das variáveis abióticas mensuradas na baía da Marambaia, baía de Sepetiba, RJ, Brasil, de maio a julho/2006.

\begin{tabular}{lccc}
\hline Variável abiótica & (Mínimo) & Mediana & (Máximo) \\
\hline Temperatura $\left({ }^{\circ} \mathrm{C}\right)$ & $(21,0)$ & 24,0 & $(28,0)$ \\
Salinidade & $(30,0)$ & 32,0 & $(36,0)$ \\
Transparência $(\mathrm{m})$ & $(0,6)$ & 1,2 & $(1,5)$ \\
Turbidez $(\mathrm{mg} / \mathrm{L})$ & $(26,7)$ & 37,7 & $(51,7)$ \\
Amônia $(\mu \mathrm{mol} / \mathrm{L})$ & $(0,7)$ & 1,8 & $(4,1)$ \\
Nitrito $(\mu \mathrm{mol} / \mathrm{L})$ & $(0,2)$ & 0,5 & $(1,1)$ \\
Nitrato $(\mu \mathrm{mol} / \mathrm{L})$ & $(1,4)$ & 2,0 & $(2,6)$ \\
Fosfato $(\mu \mathrm{mol} / \mathrm{L})$ & $(0,2)$ & 0,4 & $(1,0)$ \\
\hline
\end{tabular}

limitantes a espécie na baía da Marambaia. Os resultados sugerem que a introdução desta espécie para fins de maricultura em balsas flutuantes não está promovendo danos ambientais no local até o momento. Entretanto, devido aos resultados de sua dispersão e estabelecimento nos corais do Havaí, após cerca de três décadas da sua introdução (Russell 1983; Rodgers \& Cox 1999; Smith et al. 2002; Conklin \& Smith 2005), tanto via crescimento vegetativo quanto por esporos, assim como os relatos de bioinvasão na Índia (Bagla 2008; Chandrasekaran et al. 2008) e na Venezuela (Barrios et al. 2007) recomenda-se o monitoramento ambiental permanente dos cultivos de Kappaphycus alvarezii no Brasil.

Visando à manutenção da biodiversidade marinha, constatada a aplicabilidade da metodologia apresentada, seu uso é proposto como protocolo para monitorar o estabelecimento de Kappaphycus alvarezii fora do sistema de cultivo, na área permitida para o cultivo desta espécie, compreendida entre a baía de Sepetiba (RJ) e a Ilha Bela (SP), pela Instrução Normativa n. 185 de 23 de julho de 2008. Recomenda-se, então: a realização de vistorias trimestrais de estruturas reprodutivas em mudas cultivadas; vistorias anuais em placas acrílicas; cultivo in vitro de material fértil para verificar a sua viabilidade; vistoria e quantificação freqüente de mudas no entorno das fazendas marinhas e anualmente em áreas controle e realização de experimentos semestrais de transplantes de mudas.

\section{Agradecimentos}

À Sete Ondas Biomar, pela bolsa fornecida durante o período de realização da monografia de graduação de Beatriz Castelar e pelo apoio logístico oferecido nas atividades de campo.

\section{Referências bibliográficas}

Amado Filho, G.M.; Barros-Barreto, M.B.; Marins, B.B.V.; Felix, C. \& Reis, R.P. 2003. Estrutura da comunidade fitobentônica do infralitoral da baía de Sepetiba, RJ, Brasil. Revista Brasileira de Botânica 26: 329-342.
Ask, E. 2006. Cultivating Cottonii and Spinosum: A"how to" guide resources. In: A.T Critchley; M. Ohno \& D.B. Largo (eds.). World Seaweed Resources - An authoritative reference system. Publicado em CD Rom. Amsterdam, Expert Centre for Taxonomic Identification (ETI), University of Amsterdam.

Ask, E.I. \& Azanza, R.V. 2002. Advances in cultivation technology of commercial eucheumatoid species: a review with suggestion for future research. Aquaculture 206: 257-277.

Ask, E.I.; Batibasaga, A.; Zertuche-Gonzalez, J.A. \& San, M. 2003. Three decades of Kappaphycus alvarezii (Rhodophyta) introduction to non-endemic locations. Pp. 49-57. In: A.R. Chapman; O.R.J. Anderson; V.J. Vreeland \& I.R. Davison (eds.). Proceedings of the $17 \mathrm{th}$ International Seaweed Symposium. Cape Town 2001. Oxford, Oxford University Press.

Azanza-Corrales, R.; Mamauag, S.S.; Alfiler, E. \& Orolfo, M.J. 1992. Reproduction in Eucheuma denticulatum (Burman) Collins and Hervey and Kappaphycus alvarezii (Doty) Doty farmed in Danajon Reef, Philippines. Aquaculture 103: 29-34.

Bagla, P. 2008. Seaweed Invader Elicits Angst in India. Science 320: 1271.

Barrios, J.; Bolaños, J. \& López, R. 2007. Blanqueamiento de arrecifes coralinos por la invasión de Kappaphycus alvarezii (Rhodophyta) en Isla Cubagua, Estado Nueva Esparta, Venezuela. Boletin del Instituto Oceanografico de Venezuela 46 : 147-152.

Bulboa, C.R. \& Paula, E.J. 2005. Introduction of non-native species of Kappaphycus (Rhodophyta, Gigartinales) in subtropical waters: Comparative analysis of growth rates of Kappaphycus alvarezii and Kappaphycus striatum in vitro and in the sea in southeastern Brazil. Phycological Research 53: 183-188.

Bulboa, C.R.B.; Paula, E.J. \& Chow, F. 2008. Germination and survival of tetraspores of Kappaphycus alvarezii var. alvarezii (Solieriaceae, Rhodophyta) introduced in subtropical waters of Brazil. Phycological Research 56: 39-45.

CBD - Convention on Biological Diversity 2004. Solutions for Sustainable Mariculture - Avoiding the Adverse Effects of Mariculture on Biological Diversity. Report of the ad hoc Technical Expert Group on Mariculture. Secretariat of the Convention on Biological Diversity Technical Series N. 12.

Chandrasekaran, S.; Nagendran, N.A.; Pandiaraj, D.; Krishnankutty, N. \& Kamalakannan, B. 2008. Bioinvasion of Kappaphycus alvarezii on corals in the Gulf of Mannar, India. Current Science 94: 1167-1172.

Conklin, E.J. \& Smith, J.E. 2005. Abundance and spread of the invasive red algae, Kappaphycus spp., in Kane'ohe Bay, Hawai'i and an experimental assessment of management options. Biological Invasions 7: 1029-1039.

De Wreede, R.E. 1985. Destructive (harvest) sampling. Pp.147-160. In: M.M. Littler \& D.S. Littler (eds.). Handbook of Phycological Methods: Ecological Field Methods: Macroalgae. Cambridge, Cambridge University Press.

Doty, M.S. 1987. The production and use of Eucheuma. Pp. 123-161. In: M.S. Doty; J.F. Cady \& B. Santelices (eds.). Case studies of seven commercial seaweed resources. Rome, FAO Fisheries Tecnical Paper 281.

Eklöf, J.S.; Torre Castro, M. de la \& Adelsköld, L. 2005. Differences in macrofaunal and seagrasses assemblages in seagrasses beds and without seaweed farms. Estuarine, Coastal and Shelf Science 63: $385-396$.

FAO - Food and Agriculture Organizations of the United Nations 1996. Precautionary approach to capture fisheries and species introductions. Rome, FAO Technical Guidelines for Responsible Fisheries N.2.

Glenn, E.P. \& Doty, M.S. 1990. Growth of the seaweeds Kappaphycus alvarezii, $K$. striatum and Eucheuma denticulatum as affected by environment in Hawaii. Aquaculture 84: 245-255.

Grasshoff, K.; Ehrhardt, M. \& Kremling, K. 1983. Methods of Seawater Analysis, $2^{\text {nd }}$ ed. Berlin, Verlag Chemie. 
Mshigeni, K.E. 1979. The economic algal Eucheuma (Rhodophyta, Gigartinales): Observation on the morphology and distribution ecology of Tanzanian species. Botanica Marina 22: 437-445.

Oliveira, E.C. 1990. The rationale for seaweed cultivation in Latin America. Pp.135-141. In: E.C. Oliveira \& N. Kautsky (eds.). São Paulo, Universidade de São Paulo.

Oliveira, E.C. 2005. Considerações sobre o impacto ambiental do cultivo da alga Kappaphycus alvarezii na costa sudeste do Brasil. Boletim Ficológico, Ano 24.

Oliveira, E.C. \& Miranda, G.E.C. 1998. Aspectos sociais e econômicos da exploração de algas marinhas no Brasil. Pp. 147-156. In: E.J. Paula; M. Cordeiro-Marino; D.P. Santos; E.M. Plastino; M.T. Fujii \& N.S. Yokoya (eds.). IV Congresso Latino Americano de Ficologia, II Reunião IberoAmericana de Ficologia e VII Reunião Brasileira de Ficologia. Caxambu 1996. EXATA Ed. v.II.

Paula, E.J.; Pereira R.T.L. \& Ostini, S. 1998. Introdução de espécies exóticas de Eucheuma e Kappaphycus (Gigartinales, Rhodophyta) para fins de maricultura no litoral brasileiro: abordagem teórica e experimental. Pp. 341-357. In: E.J. Paula; M. Cordeiro-Marino; D.P. Santos; E.M. Plastino \& N.S. Yokoya (eds.). IV Congresso Latino Americano de Ficologia, II Reunião Ibero-Americana de Ficologia e VII Reunião Brasileira de Ficologia. Caxambu 1996. EXATA Ed. v.II.

Paula, E.J. \& Pereira, R.T.L. 2003. Factors affecting growth rates of Kappaphycus alvarezii (Doty) Doty ex P. Silva (Rhodophyta, Solieriaceae) in sub-tropical waters of São Paulo State, Brazil. Pp. 381-388. In: A.R. Chapman; O.R.J. Anderson; V.J. Vreeland \& I.R. Davison (eds.). Proceedings of the 17th International Seaweed Symposium. Cape Town 2001. Oxford, Oxford University Press.

Paula, E.J.; Pereira, R.T.L. \& Ohno, M. 1999. Strain selection in Kappaphycus alvarezii var. alvarezii (Solieriaceae, Rhodophyta) using tetraspore progeny. Journal of Applied Phycology 11: 111-121.

Paula, E.J.; Erbert, C. \& Pereira, R.T.L. 2001. Growth rate of the carrageenophyte Kappaphycus alvarezii (Rhodophyta, Gigartinales) in vitro. Phycological Research 49: $155-161$.

Paula, E.J.; Pereira, R.T.L. \& Ohno, M. 2002. Growth rate of the carrageenophyte Kappaphycus alvarezii (Rhodophyta, Gigartinales) introduced in subtropical waters of São Paulo State, Brazil. Phycological Research 50: 1-9.
Pereira, S.D.; Villena, H.H.; Barros, L.C.; Lopes, M.B.; Panazio, W. \& Wandeck, C. 2003. Baía de Sepetiba: caracterização sedimentar. In: IX Congresso da Associação Brasileira de Estudos do Quaternário/II Congresso do Quaternário de Países de Línguas Ibéricas/II Congresso sobre Planejamento e Gestão da Zona Costeira dos Países de Expressão Portuguesa. Recife, 2003. Publicação em CD Rom. São Paulo, Associação Brasileira de Estudos do Quaternário.

Reis, R.P.; Caldeira, A.Q.; Miranda, A.P.S. \& Barros-Barreto, M.B. 2006. Potencial para maricultura da carragenófita Hypnea musciformis (Wulfen) J.V. Lamour. (Gigartinales - Rhodophyta) na Ilha da Marambaia, Rio de Janeiro, Brasil. Acta Botanica Brasilica 20: 763-769.

Rodgers, S.K. \& Cox, E.F. 1999. The distributions of the introduced rhodophytes Kappaphycus alvarezii, Kappaphycus striatum and Gracilaria salicornia in relation to various physical and biological factors in Kane'ohe Bay, O'ahu, Hawai'i. Pacific Science 53: 232-241.

Russell, D.J. 1983. Ecology of the red imported seaweed Kappaphycus striatum on Coconut Island, Oahu, Hawai'i. Pacific Science 37: $87-107$

SEMADS - Secretaria do Estado de Meio Ambiente e Desenvolvimento Sustentável do Estado do Rio de Janeiro 2001. Bacias hidrográficas e recursos hídricos da macrorregião ambiental 2 - Bacia da baía de Sepetiba, Rio de Janeiro. Rio de Janeiro, SEMADS.

Signorini, S.R. 1980. A study of circulation in Bay of Ilha Grande and Bay of Sepetiba. Boletim do Instituto Oceanográfico 29: 41-55.

Smith, J.E.; Hunter, C.L. \& Smith, C.M. 2002. Distribution and reproductive characteristics of nonindigenous and invasive marine algae in the Hawaiian Island. Pacific Science 56: 299-315.

Starr, R.C. \& Zeikus, J.A. 1993. Utex-The culture collection of algae at the University of Texas at Austin. Journal of Phycology 29: 92-93.

Trono, G.C. 1993. Eucheuma and Kappaphycus: Taxonomy and Cultivation. Pp. 75-88. In: M. Ohno \& A.T. Chritchley (eds.). Seaweed cultivation and marine ranching. Yokosuka, Japan International Cooperation Agency (JICA).

Verlecar, X.N. \& Pereira, N. 2006. Is Kappaphycus alvarezii heading marine bioinvasion? Currente Science 90: 619-620.

Wakibia, J.G.; Bolton, J.J.; Keats, D.W. \& Raitt, L.M. 2006. Factors influencing the growth rates of three commercial eucheumoids at coastal sites in southern Kenya. Journal of Applied Phycology 18: 565-573.

Zar, J.H. 1996. Biostatistical Analysis. New Jersey, Prentice Hall. 